\title{
Acute infections, vaccination and prevention of cardiovascular disease
}

\author{
Mohammad Madjid MD
}

$\infty \quad$ See related research paper by Lamontagne and colleagues, page 773

$\mathrm{E}$ xtensive research in recent decades has established a critical role for cellular and humoral inflammation in the initiation and progression of atherosclerosis and its acute clinical presentations. ${ }^{1}$ Although atherosclerotic disease has a slow progression over many years, several intrinsic and extrinsic factors may trigger latent stable atherosclerotic plaques to become inflamed, unstable plaques that often rupture and start a cascade leading to thrombus formation and acute coronary syndromes or sudden cardiac death. Respiratory infections are among the known triggers for such acute cardiovascular events.

In the past 2 decades, many basic and epidemiologic studies have suggested a role for chronic indolent infections, such as Chlamydia pneumoniae, in the chronic progression of atherosclerotic lesions over years. Failure of clinical trials using antibiotics against $C$. pneumoniae to prevent cardiovascular events has led to a decreased interest in the role of infections in cardiovascular disease. However, in contrast to chronic infections, acute infections may cause acute coronary syndromes by triggering severe and abrupt inflammatory changes in high-risk coronary plaques over a few days or weeks. Prevention or treatment of these infections may offer new targets for coronary prevention. ${ }^{2}$

In this issue of CMAJ, Lamontagne and colleagues ${ }^{3}$ present their findings from a large hospital-based database study. The authors studied the incidence of myocardial infarctions among those who had or had not received a pneumococcal vaccine before admission to hospital. They include patients who were free of known atherosclerotic disease but who were at risk of myocardial infarction based on age (men aged 45 years or older, women aged 50 years or older) and who had at least 1 cardiovascular disease risk factor (hypertension, diabetes or hyperlipidemia). The authors report that people who had a myocardial infarction were less likely than those who did not have a myocardial infarction to have received a pneumococcal polysaccharide vaccine $(7.1 \%$ v. $11.6 \%$; adjusted odds ratio $0.53,95 \%$ confidence interval $0.40-0.70$ ).

The study by Lamontagne and colleagues does not include data on the incidence of pneumonia and its relation to myocardial infarction in cases and controls. However, it is reasonable to hypothesize that pneumococcal vaccination may protect against cardiovascular events by preventing pneumonia, as the latter has been shown to trigger myocardial infarction. Musher and colleagues ${ }^{4}$ reviewed a series of 170 patients admitted to hospital with pneumococcal pneumonia over a 5year period and found that about $7 \%$ of patients had a myocardial infarction while in hospital. They also report that about $20 \%$ of patients had either a myocardial infarction, atrial fibrillation, or new or worsening congestive heart fail-

\section{Key points}

- Infections of the upper respiratory tract, including pneumonia and influenza, can trigger acute coronary syndromes.

- Prevention of these infections by vaccination may prevent cardiovascular events in people at high risk.

- Rates of pneumococcal and influenza vaccination are below the optimal level in most countries.

- Physicians need to improve vaccination rates by actively advocating vaccination following established guidelines.

- Special attention should be paid to symptoms and signs of cardiac events in patients at high risk for cardiovascular disease who have an upper respiratory tract infection.

ure while in hospital. ${ }^{4}$ Other studies have suggested that infections in the upper respiratory tract and urinary tract can trigger myocardial infarctions. ${ }^{5}$ Spodick and colleagues reported that, in the 2-week period before being admitted to hospital for a myocardial infarction, $28 \%$ of patients had acute respiratory symptoms. ${ }^{6}$ In an autopsy-based study, my group observed that influenza epidemics are associated with a sharp rise in the number of deaths caused by myocardial infarction and ischemic heart disease. ${ }^{7}$ In fact, during almost all influenza epidemics and pandemics (except for the 1918 Spanish influenza pandemic), about twice as many people die of cardiac causes as die of pneumonia. ${ }^{8}$ Myocardial infarction was also reported to be the cause of death in 2 of 5 fatal cases among 75 patients with severe acute respiratory syndrome. ${ }^{9}$

Multiple mechanisms could contribute to the cardioprotective effect of pneumococcal vaccination. A study in mice lacking the low-density lipoprotein (LDL) receptor suggested that the molecular mimicry between epitopes of Streptococcus pneumoniae and oxidized LDL may lead to increased anti-oxidized LDL immunoglobins following pneumococcal vaccination, which may lead to a decreased extent of atherosclerosis. ${ }^{10}$ However, production of such antibodies following pneumococcal vaccination in humans has not been confirmed. ${ }^{11}$ This interesting pathway requires further research. In addition, $S$. pneumoniae and other respiratory infections may exert many acute effects directly relevant to acute coronary syndromes. My colleagues and I have previously shown that influenza infection leads to excessive recruitment of inflammatory cells to atherosclerotic aortic plaques in mice lacking apolipoprotein E receptor. ${ }^{12}$ Similarly, compared with controls, the coronary arteries of patients who died of acute systemic infections show a higher

From the Texas Heart Institute and the Baylor College of Medicine, Houston, USA 
number of macrophages and $\mathrm{T}$ cells in their adventitia and periadventitial fat, and more dendritic cells in the intima and media. ${ }^{13}$ In synergy with local cellular inflammation, there is an extensive systemic inflammation heralded by acute increase in systemic markers of inflammation. ${ }^{2}$ These acute inflammatory changes are paralleled by activation of the coagulation cascade and increased levels of coagulation factors. Finally, such acute infections are also associated with tachycardia, hemodynamic stress, fever, dehydration, increased plasma viscosity, release of endogenous cathecholamines, possible demand ischemia, severe endothelial dysfunction and qualitative pro-oxidant changes in high-density lipoprotein. Each of these can play an important role in acute coronary syndromes. ${ }^{2,14}$

Given the pivotal role of inflammation and its relation to infection in the development of acute coronary syndromes, it would be prudent to test drugs that affect inflammatory mechanisms in clinical scenarios involving atherosclerosis. Statins are known to have such anti-inflammatory effects. ${ }^{1}$ There have been conflicting conclusions from studies examining the effect of statins on mortality of people admitted to hospital with community-acquired pneumonia. New well-designed studies of statins in the context of acute infection focused on cardiovascular outcomes are needed. Preliminary reports from my laboratory suggest that statins have a direct antiinfluenza effect, ${ }^{15}$ and they could be tested for similar effects against $S$. pneumoniae and other microbial agents. Hypothetically, in adults with respiratory infections, acetylsalicylic acid (ASA) could be useful not only for relief of respiratory symptoms but also for preventing coronary events, because ASA may help to reduce inflammation and inhibit platelet function during high-risk periods. ${ }^{2}$ Finally, further research is needed to assess whether the use of antimicrobials can prevent cardiovascular events if used in a timely manner when infections occur without or despite vaccination.

Multiple case-control, cohort and randomized clinical trials in different settings have shown that preventing influenza by vaccination can reduce the risk of recurrent myocardial infarction, sudden cardiac death, cardiac hospital admissions, need for revascularization and stroke. ${ }^{2,14}$ The current study by Lamontagne and colleagues suggests that pneumococcal vaccination could similarly be a safe and inexpensive tool to prevent cardiovascular events. However, results of case-control studies should be interpreted with caution. "Healthy user" bias is a possibility in case-control studies. Receipt of pneumococcal vaccine may be an indicator of better access to health care, healthier life style and better control of risk factors. The study findings were significant after adjustment for several confounders; however, statistical models may not completely overcome the complex interactions between confounders. Further prospective and randomized studies are needed to confirm these findings.

Until confirmatory studies are available, clinicians should comply with available guidelines by increasing current vaccination rates among patients at high risk. In the United States, rates of pneumococcal and influenza vaccination are well below the established goals, and the situation is worse in many other countries. In addition, clinicians should reduce the baseline risk of cardiovascular events in vulnerable patients by judicious use of antihypertensive, lipid-lowering drugs (preferably statins) and ASA.

Competing interests: Mohammad Madjid received an honorarium from Pfizer for participation in an advisory board meeting in 2007.

\section{REFERENCES}

1. Willerson JT, Ridker PM. Inflammation as a cardiovascular risk factor. Circulation 2004;109(Suppl 1):II2-10

2. Madjid M, Naghavi M, Litovsky S, et al. Influenza and cardiovascular disease: a new opportunity for prevention and the need for further studies. Circulation 2003;108:2730-6.

3. Lamontagne F, Garant MP, Carvalho JC, et al. Pneumococcal vaccination and risk of myocardial infarction. CMAJ 2008;179:773-7.

4. Musher DM, Rueda AM, Kaka AS, et al. The association between pneumococcal pneumonia and acute cardiac events. Clin Infect Dis 2007;45:158-65.

5. Smeeth L, Thomas SL, Hall AJ, et al. Risk of myocardial infarction and stroke after acute infection or vaccination. N Engl J Med 2004;351:2611-8.

6. Spodick DH, Flessas AP, Johnson MM. Association of acute respiratory symptoms with onset of acute myocardial infarction: prospective investigation of 150 consecutive patients and matched control patients. Am J Cardiol 1984;53:481-2.

7. Madjid M, Miller CC, Zarubaev VV, et al. Influenza epidemics and acute respiratory disease activity are associated with a surge in autopsy-confirmed coronary heart disease death: results from 8 years of autopsies in 34,892 subjects. Eur Heart J 2007;28:1205-10.

8. Madjid M, Casscells SW. Of birds and men: cardiologists' role in influenza pandemics. Lancet 2004;364:1309.

9. Peiris JS, Chu CM, Cheng VC, et al. Clinical progression and viral load in a community outbreak of coronavirus-associated SARS pneumonia: a prospective study. Lancet 2003;361:1767-72.

10. Binder CJ, Horkko S, Dewan A, et al. Pneumococcal vaccination decreases atherosclerotic lesion formation: molecular mimicry between streptococcus pneumoniae and oxidized LDL. Nat Med 2003;9:736-43.

11. Nguyen JT, Myers N, Palaia J, et al. Humoral responses to oxidized low-density lipoprotein and related bacterial antigens after pneumococcal vaccine. Transl Res 2007;150:172-9.

12. Naghavi M, Wyde P, Litovsky S, et al. Influenza infection exerts prominent inflammatory and thrombotic effects on the atherosclerotic plaques of apolipoprotein e-deficient mice. Circulation 2003;107:762-8.

13. Madjid M, Vela D, Khalili-Tabrizi H, et al. Systemic infections cause exaggerated local inflammation in atherosclerotic coronary arteries: clues to the triggering effect of acute infections on acute coronary syndromes. Tex Heart Inst J 2007;34:11-8.

14. Madjid M, Aboshady I, Awan I, et al. Influenza and cardiovascular disease: Is there a causal relationship? Tex Heart Inst J 2004;31:4-13.

15. Haidari M, Ali M, Casscells W, et al. Statins block influenza infection by downregulating Rho/rho kinase pathway [abstract]. Circulation 2007;116:II_7.

Correspondence to: Dr. Mohammad Madjid, Texas Heart Institute, 6770 Bertner Ave., MC 2-255, Houston TX 77030, USA; fax832 355-9595; mmadjid@gmail.com 